\title{
Novel Polarization-Agile Annular Waveguide Slot Antennas
}

\author{
Siamak Ebadi and Keyvan Forooraghi \\ Microwave Lab., Tarbiat Modares University \\ Tehran, Iran
}

\section{Introduction}

Slot array antennas using rectangular waveguides were widely used in past decades based on their various important capabilities in microwave telecommunications. Having a low profile, ability to implement different current distributions and high power handling are among the advantages of such antennas. On the other hand, there exist some restrictions in optimum and efficient performance of rectangular slotted waveguide antennas due to the use of rectangular waveguides and slots in a circular boundary (Elliott, 2003). These limitations are mainly caused by the disharmony between the rectangular geometry of radiating elements and the circular boundary of the antenna. This has been the inspiration for exploring novel alternative structures with improved geometrical balance with the circular boundary (Fusco et al., 2003, Noghanian et al., 2001, Takahashi et al., 1995, Kelly et al., 1961).

Annular Waveguide Slot Antennas (AWSA) were introduced in (Ebadi et al., a, 2007) intending the application in circular boundary planar slot array antennas. Resonant characteristic along with linear polarization perpendicular to the slot direction were proved the single slot case and it was shown that the AWSA could be modeled as a shunt conductance in the annular line. As the first step to the study of an AWSA, the annular waveguide was compared with the rectangular waveguide (Ebadi et al., b, 2008) and the design procedure was provided in (Ebadi et al., c \&d, 2008). It was shown that such waveguide bend could be considered as a transmission line along the cylindrical $\varphi$ direction. Sinusoidal field distribution along the waveguide cross section and single mode transmission were also provided. As the second step, the Green's functions required for the analysis of an AWSA by the method of moment were derived and presented in (Ebadi et al., e, 2009). These functions will be used in analyzing the AWSA to derive exact field distribution along the slot and the variation of slot conductance in different frequencies.

In an AWSA, the waveguide and the slots are all circularly oriented to fit the boundary. Due to its similarity with the rectangular slotted waveguide case, the circuit model for the AWSA was proposed to be a shunt admittance. Fig. 1 shows a sample AWSA structure. The transmission line is supposed to be the H-plane waveguide bend which is also called azimuthal waveguide. The waveguide is fed from one end and is short-ended at the other 
side operating in the resonance mode. The slot length is selected as the resonance length appropriate to its radial offset from the centerline.
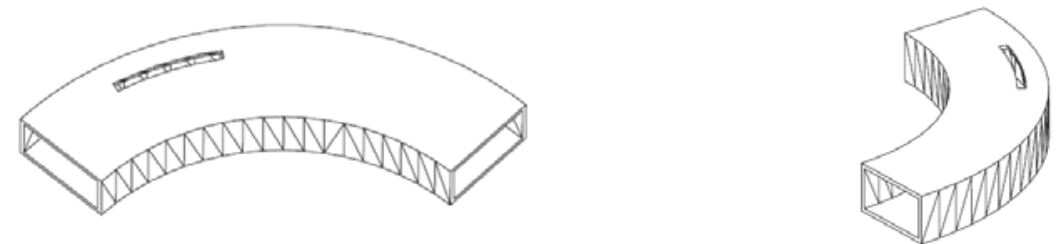

Fig. 1. Different views of an AWSA. The waveguide is an H-plane bend and the wave is annularly guided through it. (Ebadi et al., a, 2007)

Regarding the interesting applications of AWSA arrays, the main point to mention will be the polarization agility. As it was presented in (Ebadi et al., f, 2008), a four element AWSA array (four quadrants in the general form) could be used to form linear horizontal or vertical polarization. This will require four phase shifter units with two states of 0 and 180 degree (1bit phase shifters). Such polarization agility will allow the system designer to switch between the two principal polarizations in situations where one has more advantages over the other one. This characteristic is not a common property in usual antennas and is usually difficult to achieve. It was also recently reported in (Ebadi et al., g, 2009) that this property could be extended to circular polarization using phase shifters with 4 states ( 2 bits). Although the change in polarization was shown in these papers, there were lack of enough reasoning and theoretical basis. Additionally, the presented radiation patterns for each polarization did not follow a predefined and predicted shape and suffered from high levels of grating lobes.

In this chapter, detailed study of polarization agility achievement in AWSA will be presented. All required phase distribution to change the polarization to any of linear horizontal/vertical or right/left hand circular will be presented and verified by various simulations. The graphical demonstration of different phase combinations over the slots leading to different polarizations will be added. It will lead to better understanding of the polarization change concept. As another important parameter, the proper array factor will be derived for the two principal orthogonal planes which could predict the shape of the radiating pattern. This is based on the equivalent inter-element spacing between the slots in orthogonal directions. The structure will be modeled as a four-element array and the pattern could be derived theoretically. The simulation results for the radiation pattern will be compared to the presented theory and good agreement will be observed. This will be the basis for the design of more complex AWSA arrays in the future with the capability to synthesize desired $\varphi$-symmetric patterns with low level of quantization error.

\section{Analysis of a single slot AWSA (Ebadi et al., a,b,c,d,e)}

In this section a short review of the procedure for analyzing an AWSA structure will be presented. At the first step, an annular waveguide will be studied as a transmission line and some brief results of this study will be provided following by the method of moment analysis results at the end. Fig. 2 shows the proposed annular waveguide and compares it with a common rectangular one. After a set of formulations which could be followed in 
(Ebadi et al., b, 2008), a sample graphical solution of the deterministic equation for the Xband annular waveguide is provided in Fig. 3. It is observed that this waveguide completely supports the X-band in its single mode operation. Fig. 4 depicts the range of single mode operation for different values of waveguide inner radius. It is interestingly found that this structure will support the desired band for approximately all values of $a$.

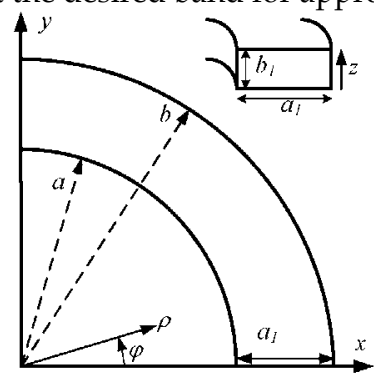

(a)

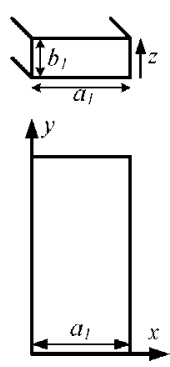

(b)

Fig. 2. Demonstration of (a) an annular waveguide and its comparison with (b) the rectangular case in the same geometry: top and 3-D views. (Ebadi et al., b, 2008)

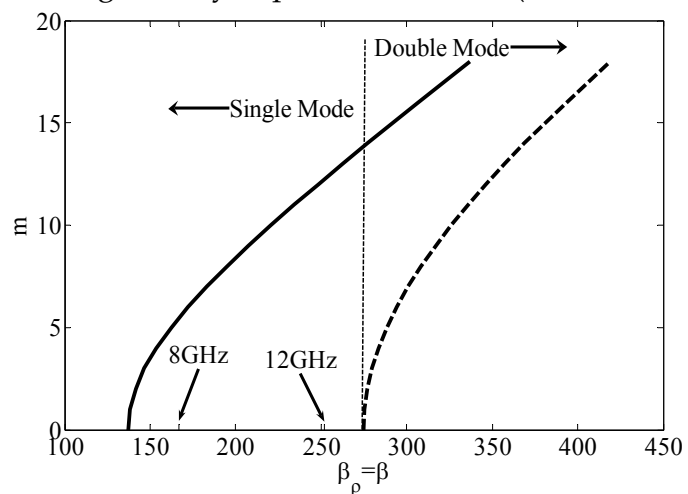

Fig. 3. Graphical solution of the deterministic equation. The region supporting the single mode transmission completely covers the X-band. (Ebadi et al., b, 2008)

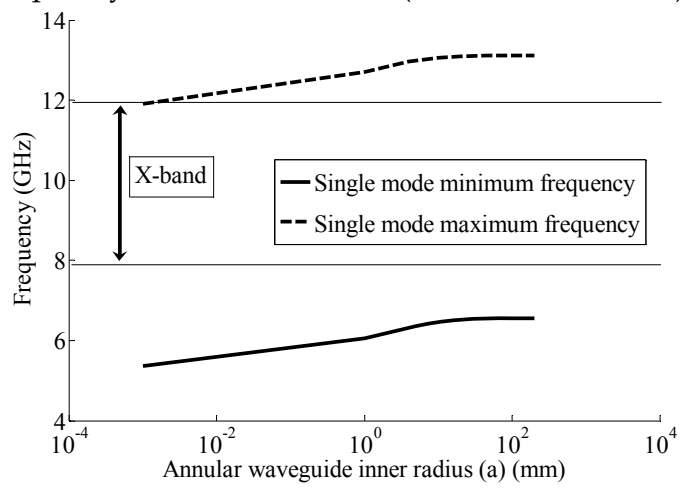

Fig. 4. Supported frequency range in the single mode operation for different values of annular waveguide inner radius $(b-a=0.9 \mathrm{in}=22.86 \mathrm{~mm})$. (Ebadi et $\mathrm{al} ., \mathrm{b}, 2008$ ) 
Effect of waveguide radius on the sinusoidal field distribution across the annular waveguide (T) is depicted in Fig. 5. It is shown that for $a>50 \mathrm{~mm}$ at X-band, this variation will be sinusoidal. The same distribution could be derived from theoretical MoM analysis and the comparison is made in Fig. 6. The other important result of such analysis would be the annular resonant length of the slot which is provided in Fig. 7. Good agreement is observed between the direct simulation and theoretical analysis in the last two diagrams.

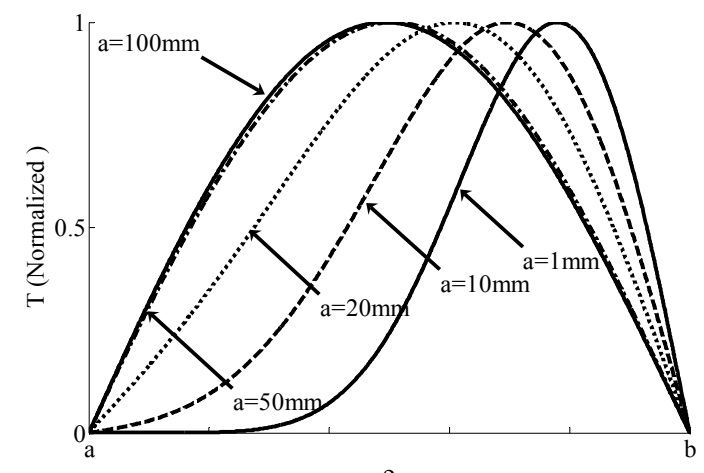

Fig. 5. Plots of $\mathrm{T}$ for different values of $a$ and for $\mathrm{m}=8$ and $\mathrm{b}-a=22.86 \mathrm{~mm}$ : for $a>30 \mathrm{~mm}$, sinusoidal field distribution becomes approximately valid. (Ebadi et al., b, 2008)

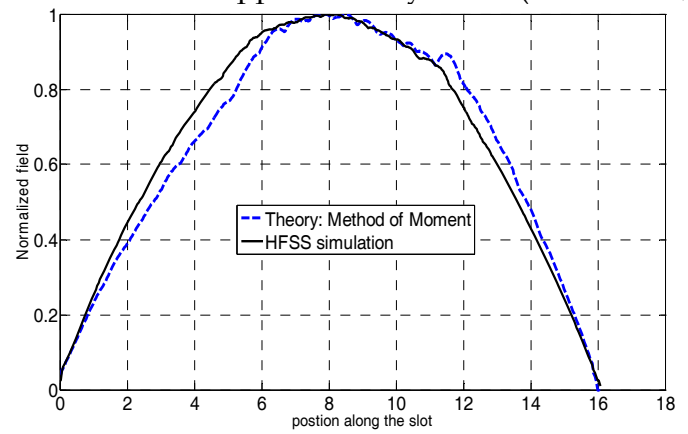

Fig. 6 Field distribution along the slot; comparison of MoM analysis and simulation

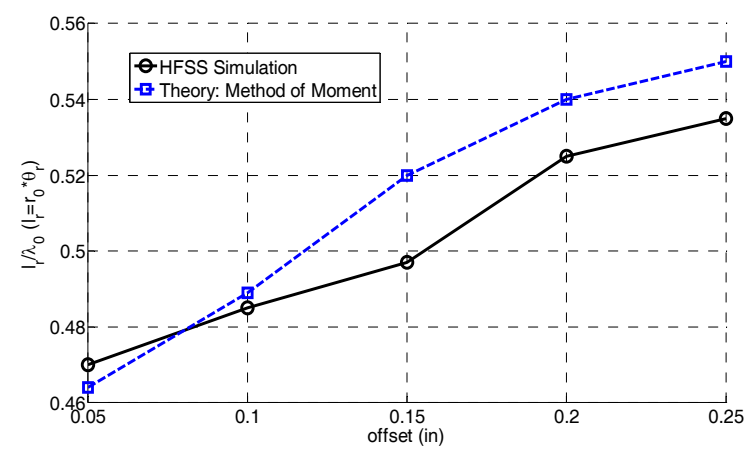

Fig. 7 Annular slot resonant length; comparison of MoM and simulation. 
Using the provided theoretical basis and based on the circuit model derived from the MoM analysis and the propagating mode in the annular waveguide described in the previous sections, a single slot AWSA was designed and simulated at $9.3 \mathrm{GHz}$. The designed antenna is fabricated as shown in Fig. 8. The impedance measurement results are provided in Fig. 9 and compared with the circuit model theory (which is that of a shunt conductance resonating at the design frequency) and simulation. The relative radiation pattern measurement results are also provided in Fig. 10 at two principal planes. Good agreement is observed between the design and measurement results.

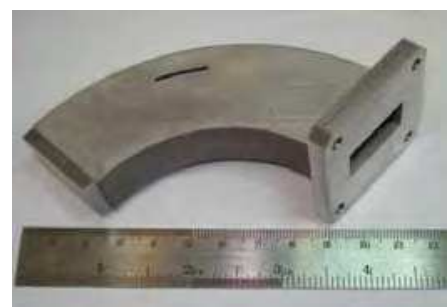

Fig. 8 The implemented AWSA structure: It is implemented through exact aluminum wirecut procedure of each face and final assembly step. (Ebadi et al., a, 2007)

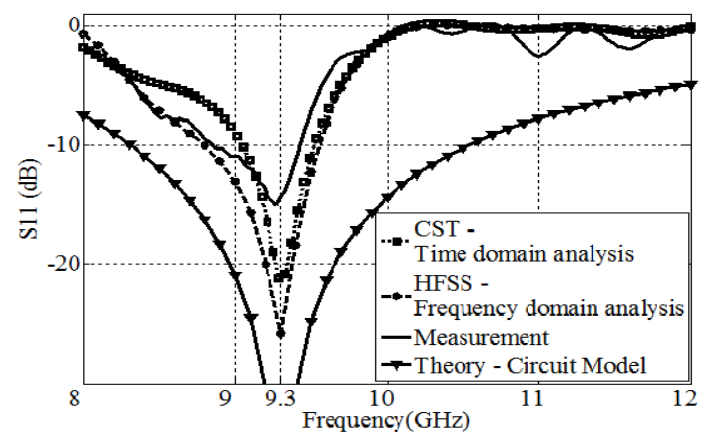

Fig. $9 S_{11}$ of the implemented AWSA. Circuit model, full wave simulations and measurement results are compared. (Ebadi et al., a, 2007)

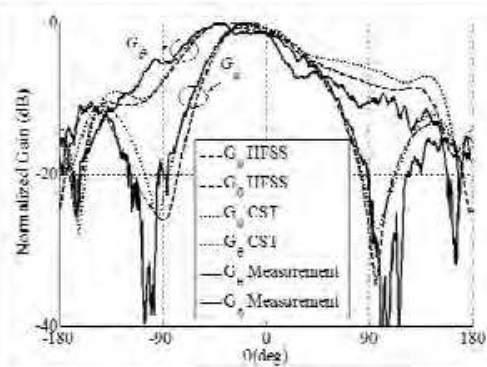

(a)

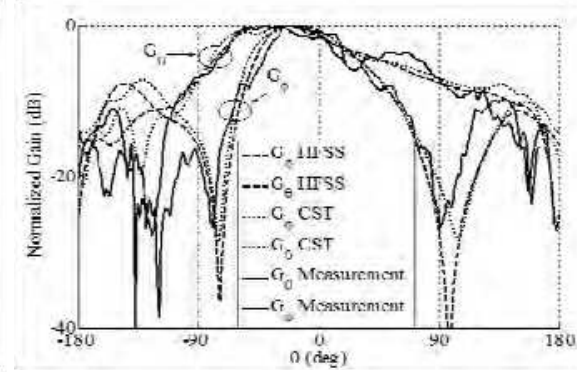

(b)

Fig. 10 Normalized pattern of the proposed AWSA: (a) $\varphi=0$ (b) $\varphi=90$ (Ebadi et al., a, 2007) 


\section{Application: polarization agility in AWSA (Ebadi et al., $\mathrm{f} \& \mathrm{~g}$ )}

\subsection{Theoretical background}

In this section, the procedure for designing an array of the proposed AWSA with the ability to change the polarization using a set of phase shifters will be introduced. The array under study is a four element (four quadrants in its general form). Each element consists of an annular waveguide with an annular slot on its top face. The waveguides are closed at both ends and are excited by some coupling slots or coaxial lines from the bottom. In this chapter we do not care about the annular waveguide excitation details and it is supposed to be done ideally. The top view of the array is depicted in Fig. 11(a). As it is shown, the total antenna is symmetric regarding both principal planes $(x=0$ and $y=0)$. The four waveguides are excited in the same way and there are the same current distributions on the slots.

As known from the slot theory, the electric field in the slots will mainly have the cylindrical $\rho$ component. It neglects the components of electric field along the slot direction $(\varphi)$. Assuming narrow slot width the main electric field component over the slots could be divided to two components along $\mathrm{x}$ and $\mathrm{y}$ as follows

$$
\left\{\begin{array}{l}
\overrightarrow{E_{r 1}}=\hat{x} E_{x}+\hat{y} E_{y} \\
\overrightarrow{E_{r 2}}=-\hat{x} E_{x}+\hat{y} E_{y}
\end{array}, \quad\left\{\begin{array}{l}
\overrightarrow{E_{r 3}}=-\hat{x} E_{x}-\hat{y} E_{y} \\
\overrightarrow{E_{r 4}}=\hat{x} E_{x}-\hat{y} E_{y}
\end{array}\right.\right.
$$

The components have the same amplitude but since they are directed to different directions, the signs are different. These four tangential electric fields could be considered as secondary sources $(\vec{M})$ and will be responsible for the radiation of the array antenna. After insertion of the excitation coefficients (A to D) to all quarters (1 to 4 ), the far-field radiation pattern could be generally defined as in (2).

$$
\left\{\begin{array} { l } 
{ \vec { E _ { 1 } ^ { r a d } } = f ( A \{ \hat { x } E _ { x } + \hat { y } E _ { y } \} ) } \\
{ \vec { E _ { 2 } ^ { r a d } } = f ( B \{ - \hat { x } E _ { x } + \hat { y } E _ { y } \} ) } \\
{ \vec { E _ { 3 } ^ { r a d } } = f ( C \{ - \hat { x } E _ { x } - \hat { y } E _ { y } \} ) } \\
{ \vec { E _ { 4 } ^ { r a d } } = f ( D \{ \hat { x } E _ { x } - \hat { y } E _ { y } \} ) }
\end{array} \rightarrow \left\{\begin{array}{l}
\overrightarrow{E_{T}^{r a d}}=\hat{x} E_{T x}^{r a d}+\hat{y} E_{T y}^{r a d} \\
E_{T x}^{r a d}=f\left(\{A-B-C+D\} E_{x}\right) \\
E_{T y}^{r a d}=f\left(\{A+B-C-D\} E_{y}\right)
\end{array}\right.\right.
$$

In which $\overrightarrow{E_{1}^{r a d}}$ to $\overrightarrow{E_{4}^{r a d}}$ represent the radiated fields due to the four slots and $\overrightarrow{E_{T}^{\text {rad }}}$ represents the total radiated field which is the weighted combination of the four aforementioned components. The function $\mathrm{f}$ will relate the far-field radiated electric field components to the four magnetic currents on the slots based on the half space Green's function. It should be mentioned that the field components under study could also be chosen as $E_{\theta}$ and $E_{\varphi}$ without loss of generality.

It is clearly obvious that the total electric field components are dependent on the respective feed excitation of the annular waveguides in each quarter. This will be the motivation to form array configurations with proper quarter feed excitations to achieve desired total 
polarizations. Although this proportional relation could be complicated radiating functions in general, but due to the structure symmetry and the proposed assumptions, the relation will be shown to be considerably valid through detailed simulation for different cases.

As a demonstration, Fig. 11 (b) shows the resulted electric field components for the three cases of null radiation, linear horizontal and linear vertical polarizations. It also shows the effective distance between the slots considering them as single point sources. In the topright case, it will be found that if the waveguides are excited with the same phase distributions, the four components will cancel each other and there will be null radiation in the broadside direction. In the two bottom figures it is shown in this figure how the electric field components will rotate with simple $0^{\circ}$ or $180^{\circ}$ phase shifts in a way that the total radiated field vector will point to our desired horizontal or vertical directions. As mentioned before, there are four 1-bit phase shifters (two states for each) required to change the polarization to these cases in the linear form.

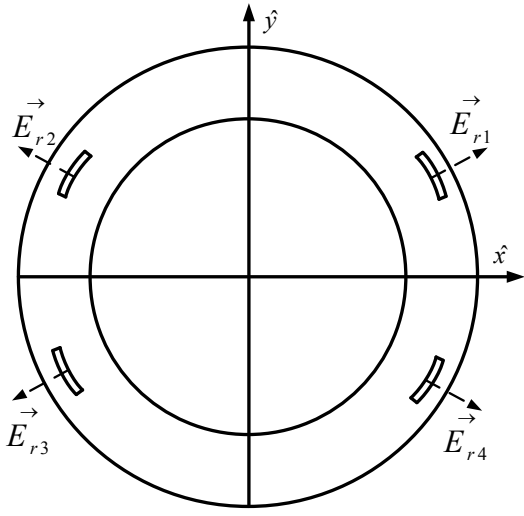

(a)

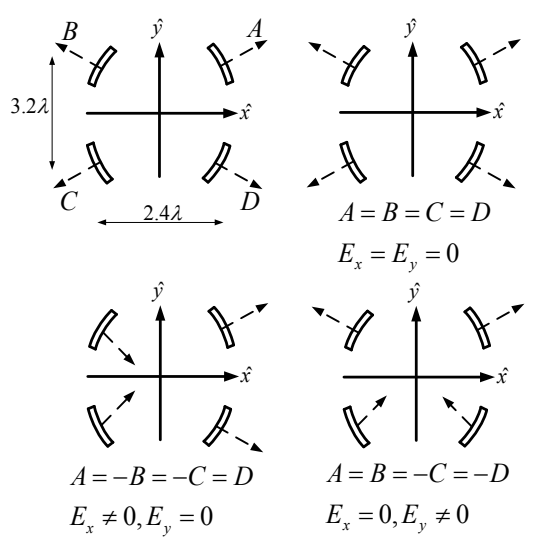

(b)

Fig 11. A 4-element AWSA array. (a) the combination of four short-ended single slot AWSAs (b) corresponding electric field components for null and linear horizontal/vertical radiations.

Table I shows the proposed values for feed excitation coefficients in order to achieve linear horizontal/vertical polarization in as well as LHCP or LHCP. In the first column the four possible radiation cases are listed along with the case of no radiation. In the second column the required phase distribution for each case are provided and the resulted field components are listed in the third column. For the circular cases, both $\mathrm{x}$ and $\mathrm{y}$ components of the total radiated electric field should be present with $90^{\circ}$ of phase shift lead/lag based on the kind of circular polarization. It is interestingly observed that by simple $180^{\circ}$ or $90^{\circ}$ phase shifts at three quarters with respect to the reference one, the total electric field could have the desired polarization. 


\begin{tabular}{|c|c|c|}
\hline Desired Polarization & $\begin{array}{c}\text { Feed Coefficients } \\
\text { Requirements }\end{array}$ & Resulted Field Vectors \\
\hline $\begin{array}{l}\text { Zero Electric Field } \\
\text { (Null radiation) }\end{array}$ & $A=B=C=D$ & $E_{T x}^{r a d}=E_{T y}^{r a d}=0$ \\
\hline Linear (y) & $\begin{array}{l}B=A \\
C=-A \\
D=-A\end{array}$ & $\begin{array}{l}E_{T x}^{r a d}=0 \\
E_{T y}^{r a d}=4 A E_{y}\end{array}$ \\
\hline Linear $(x)$ & $\begin{array}{l}B=-A \\
C=-A \\
D=A\end{array}$ & $\begin{array}{l}E_{T x}=4 A E_{x} \\
E_{T y}=0\end{array}$ \\
\hline LHCP & $\begin{array}{l}B=j A \\
C=-A \\
D=-j A \\
\left(E_{x}=E_{y}\right)\end{array}$ & $\begin{array}{l}E_{T x}^{r a d}=2 A(1-j) E_{x}=2 \sqrt{2} A E_{x} e^{-j \frac{\pi}{4}} \\
E_{T y}^{r a d}=2 A(1+j) E_{x}=2 \sqrt{2} A E_{x} e^{+j \frac{\pi}{4}}\end{array}$ \\
\hline RHCP & $\begin{array}{l}B=-j A a \\
C=-A \\
D=j A \\
\left(E_{x}=E_{y}\right)\end{array}$ & $\begin{array}{l}E_{T x}^{r a d}=2 A(1+j) E_{x}=2 \sqrt{2} A E_{x} e^{+j \frac{\pi}{4}} \\
E_{T y}^{r a d}=2 A(1-j) E_{x}=2 \sqrt{2} A E_{x} e^{-j \frac{\pi}{4}}\end{array}$ \\
\hline
\end{tabular}

Table 1. Proposed Feed coefficients for the AWSA array combination: by proper choice of the coefficients, the total structure will radiate the desired polarization

\subsection{Simulation results}

A full-wave simulation based on finite-element method is performed using Ansoft HFSS to verify the proposed method for achieving arbitrary polarization in AWSA arrays. The simulated structure is shown in Fig. 12. The 4-element array is constructed using four sets of single slot AWSA as designed and measured in (Ebadi et al., a, 2007) at 9.3GHz. The four waveguides are closed at both ends and are excited from the bottom and are internally separated. There will be four available ports for the simulation of this structure with their respective phases playing a critical role in our application.

Fig. 13 depicts the simulated gain components of a single slot AWSA. As its unique property, the single slot radiation patterns have both horizontal and vertical components $\left(G_{x}\right.$ $\& G_{y}$ ) with approximately equal maximum values in both planes. This is different from the conventional rectangular slotted waveguides in which each slot has only one main gain component forcing the total array to have the same polarization as a result. As mentioned 
before, this interesting characteristic in AWSA is the motivation to form array combinations with desired arbitrary polarization.

Fig. 14 shows the radiated gain components of the 4-element array with the feed coefficients chosen from Table I to have only horizontal polarization $\left(G_{x}\right)$. It is observed that the fourelement array has only the predicted $G_{x}$ component with the $G_{y}$ component being completely cancelled down to $-300 \mathrm{~dB}$ which is quite in agreement with our prediction. It should be mentioned that the level of such cancelation will depend on mechanical considerations and the precision of the phase shifters and in actual cases will not go below $-50 \mathrm{~dB}$. The plots are shown for both principal planes $(\varphi=0,90)$.

The same simulation was performed for the linear vertical polarization $\left(G_{y}\right)$ case and is depicted in Fig. 15. As predicted, the gain component along the y direction is conserved and the other unwanted component is completely cancelled. The same discussion is valid for the level of cancelation as in the previous case. This finishes the first part of simulation results dealing with linear polarization agility and proved the proposed method in this level.

The other interesting property of an AWSA array would be the circular polarization with the right or left hand sense of rotation changeability. This would be also performed following the coefficients introduced in Table I. In this case $90^{\circ}$ phase shifts at the quarters would be also required. This will add a higher level of complexity to the structure. In this case there will be three 2-bit phase shifters required to set the desired phase differences between quarter $\mathrm{A}$ and other quarters. But this extra complexity will lead to a very interesting extra performance of the AWSA array: the designer could change the polarization of the array to circular right/left hand. This will be so useful in operating environments causing high level of rotation in the polarization of the travelling electromagnetic field.

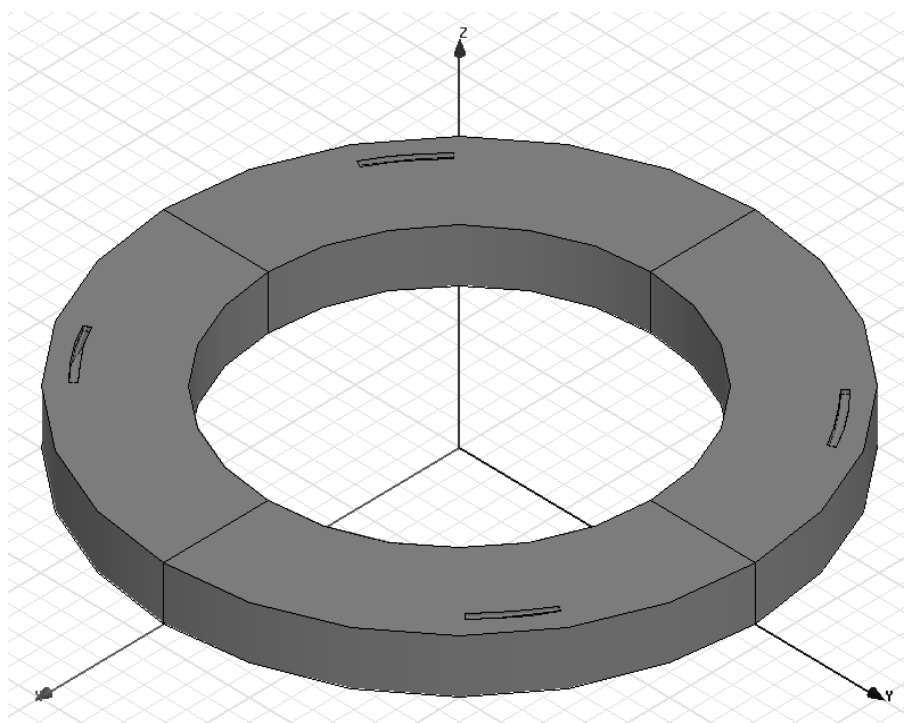

Fig 12. HFSS Simulated 4-element AWSA array. (Ebadi et al., $\mathrm{f} \& \mathrm{~g}$ ) 


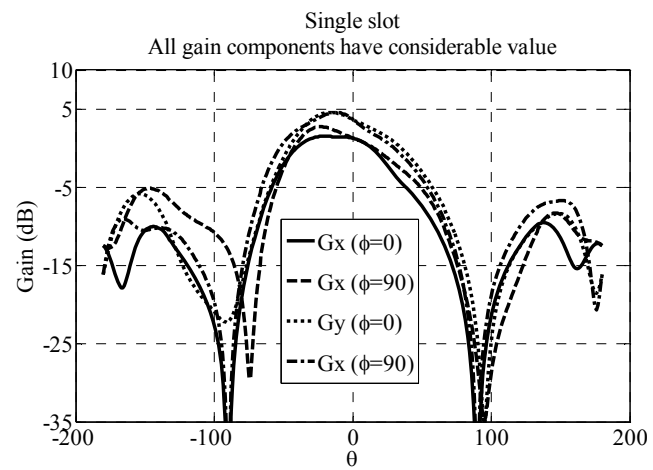

Fig. 13. Simulated pattern of a single slot (AWSA): the pattern has vertical and horizontal components as the motivation to form arrays with desired polarization. (Ebadi et al., a, 2007)

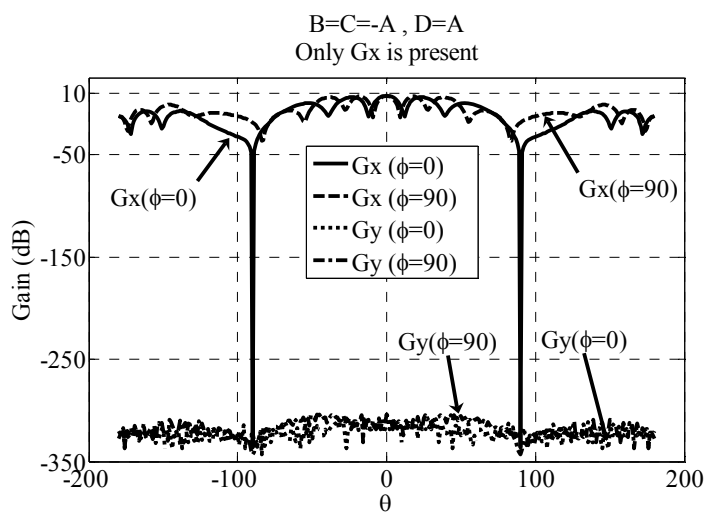

Fig. 14. Simulated radiation pattern of a 4-element AWSA array: excitation coefficients are chosen in a way to have only linear horizontal $\left(G_{x}\right)$ gain component. (Ebadi et al., $\mathrm{f} \& \mathrm{~g}$ )

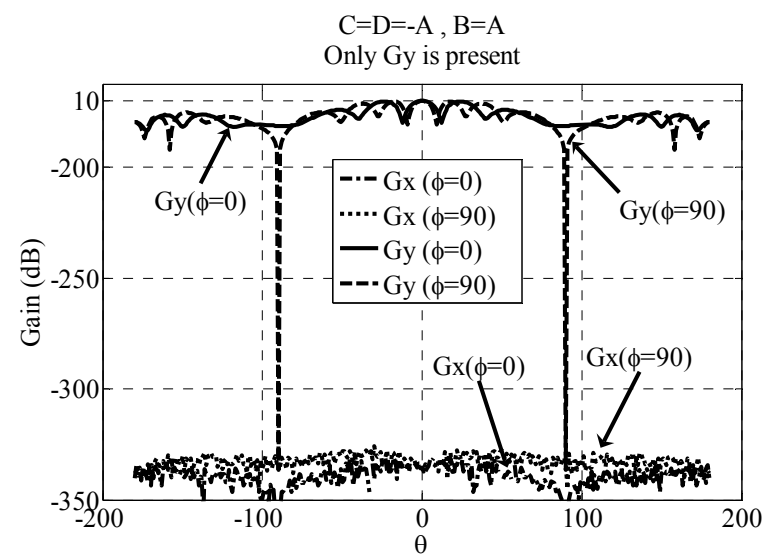

Fig. 15. Simulated radiation pattern of a 4-element AWSA array: excitation coefficients are chosen in a way to have only linear vertical $\left(G_{y}\right)$ gain component. (Ebadi et al., $\mathrm{f} \& \mathrm{~g}$ ) 
Fig. 16 shows the simulated axial ratio for left hand case (LHCP). It is observed that the antenna radiates a circular polarization (Axial ratio=1) in the region $-50<\theta<50$ which is acceptable for many practical systems. It is understood that the structure is more sensitive in circular mode and has a narrower range of operation compared to the linear case which was valid for almost all $\theta$ values. The extension of this operating region and study of different effective parameters in this behavior will be one of the future possible research interests of the authors. Fig. 17 depicts the polarization ratio for the desired LHCP case. This parameter is representative of the respective strength of left and right hand components of the circular polarization. As expected, the LHCP has a complete superiority in the aforementioned region over RHCP and the proposed method is again verified in predicting the sense of rotation in circular case. It should be mentioned however that the polarization ratio has some variations that means a small change in the level of purity of the desired polarization. Although it might be considered as another drawback of the approach, this variation is not so high and destructive.

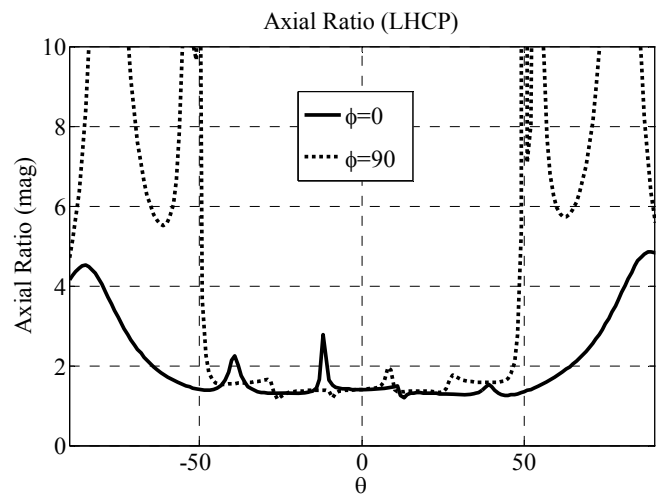

Fig. 16. Simulated axial ratio of a single slot (AWSA) in LHCP case: it is observed that the array radiates a circular polarization in a wide range of angles. (Ebadi et al., g, 2009)

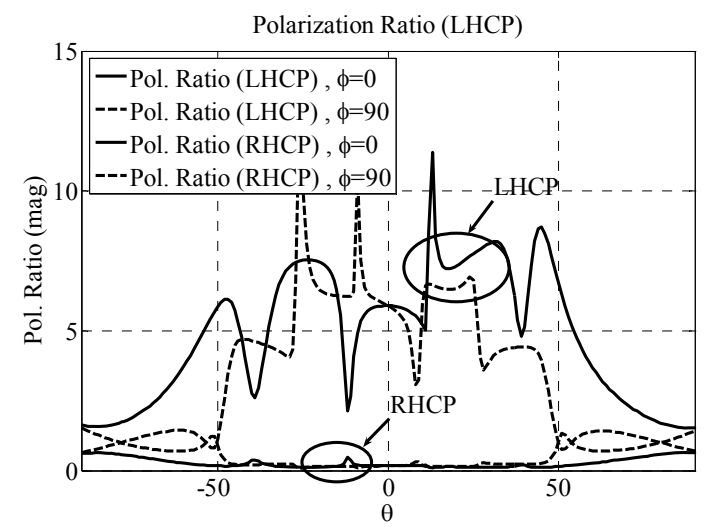

Fig. 17. Simulated polarization ratio of a 4-element AWSA array: the excitation coefficients are chosen in a way to have only LHCP and the RHCP is suppressed. (Ebadi et al., g, 2009) 
The same procedure was performed for the RHCP case. Fig. 18 proofs the circular polarization validity over the same region and Fig. 19 shows that the correct sense of rotation is achieved. Therefore, it was shown that by simple phase shifts at the four quarters, all possible practical polarizations could be achieved from the novel AWSA array

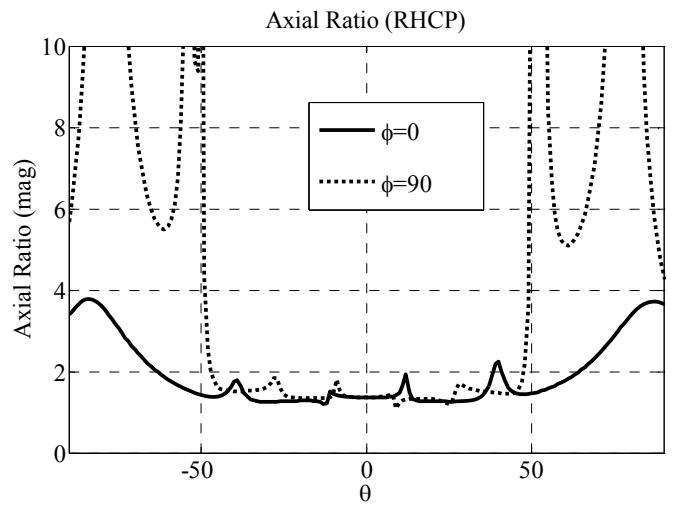

Fig. 18. . Simulated axial ratio of a single slot (AWSA) in RHCP case: it is observed that the array radiates a circular polarization in a wide range of angles. (Ebadi et al., g, 2009)

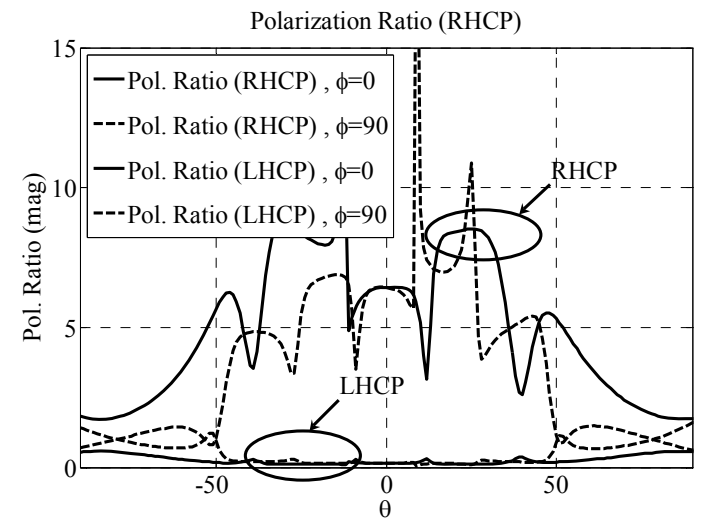

Fig. 19. Simulated polarization ratio of a 4-element AWSA array: the excitation coefficients are chosen in a way to have only RHCP and the LHCP is suppressed. (Ebadi et al., g, 2009)

\subsection{Array Factor Modeling}

Till now it was shown that by proper choice of quadrant excitations in a four element AWSA array the polarization of final radiated field could be changed between linear and circular form of any kind. The other important concept in the antenna design would be the shape of the radiation pattern. Although we have succeeded in forcing the radiated field to have our desired direction or polarization, the variation of the field in different angles in its far-field region should be studied and synthesized as well. In this section we will propose a simple method to study and predict the form of AWSA radiation pattern. 
Referring to the radiation patterns in the linear case as an example (Fig. 14 and Fig. 15), it could be observed that the patterns suffer from high level of grating lobes with almost the same values as the main beam. The reason for such behavior could be found by considering the AWSA array as a four element array with the distances between the elements provided in Fig. 11(b) in both directions. The pattern of this array could be then predicted by using array factor theory and the element patterns derived in previous sections at both planes. The equivalent arrays at both principal planes are formed and shown in Fig. 20. Using this configuration, one could predict the radiation pattern easily.

Fig. 21 compares the patterns derived from the proposed array-factor modeling method with that of full-wave simulations. It is observed that the two results are in a very good agreement in both orthogonal planes. This opens a broader field of future research and study on the concept of pattern synthesis in AWSA arrays. The four-element array studied in this chapter was proved to be successful in altering the polarization but fails to provide a low sidelobe level pattern due to the large spacing between the elements. Using arrays with larger number of elements and performing an appropriate pattern synthesis will improve the pattern behavior.

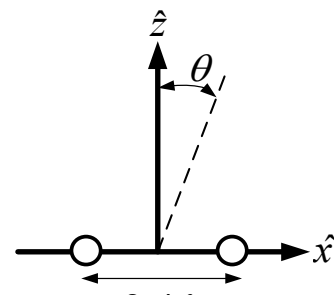

$2.4 \lambda$

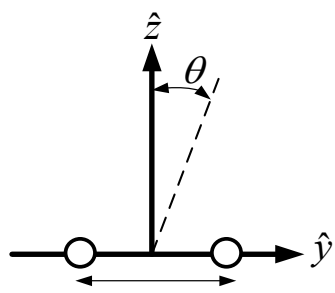

$3.2 \lambda$

Fig. 20. Two equivalent arrays of the AWSA array in Fig. 2. The radiation patterns could be theoretically predicted using these arrays.

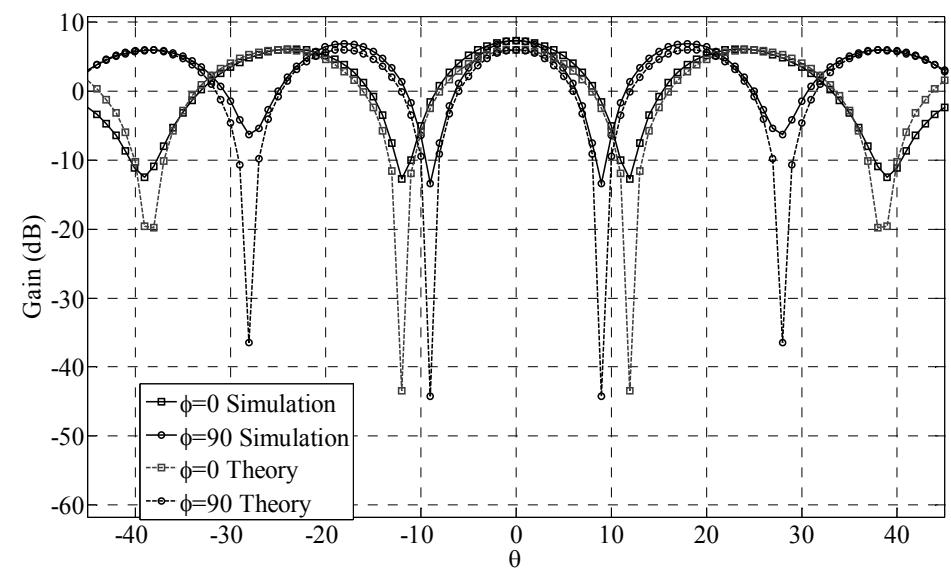

Fig. 21. Radiation pattern for linear polarization (x-direction) for two orthogonal planes: comparison of simulation and array factor theory. 


\section{Conclusion}

Annular waveguide slot antennas were introduced to form arrays with the possibility to alter the polarization of the antenna between the four major cases of linear horizontal or vertical and right or left hand circular. This important achievement needs the array to be formed in a four-quadrant scheme with three phase shifter units to set the desired phase differences with respect to the first quarter. Different phase distributions were provided for all polarization cases. The angular range in which the polarization follows the predicted form was studied for different cases and it was shown that the linear polarization is valid in a larger angular range. The radiation pattern was also theoretically predicted by modeling the array and forming the respective array factor and good agreement was achieved. Such polarization-agile AWSA array would be of high usage in complicated applications.

\section{References}

Elliott, R. S. (2003). Antenna Theory and Design, John Wiley \& Sons, Inc., New Jersey.

Fusco, V.F. \& Subbarao, B. (2003). Probe-fed circularly polarized Monopulse radial line slot antenna, Electronics Letters, Volume 39, Issue 21, pp.1495 - 1496.

Noghanian, S. \& Shafai, L. (2001). Analysis of conducting ring and double layer dielectric loaded annular slot array antennas, Microwaves, Antennas and Propagation, IEE Proceedings Volume 148, Issue 5 Page(s):327 - 333.

Takahashi , M.; Ando, M.; Goto, N.; Numano, Y.; Suzuki, M.; Okazaki, Y. \& Yoshimoto, T. (1995). Dual circularly polarized radial line slot antennas, Antennas and Propagation, IEEE Transactions on Volume 43, Issue 8, Page(s):874 - 876

Kelly, K. \& Goebels, F. (1961). Arbitrary polarization from annular slot planar antennas, Antennas and Propagation, IEEE Transactions on Volume 9, Issue 4, pp. 342 - 349.

(a) Ebadi, S. \& Forooraghi, K. (2007). An annular waveguide slot antenna, IEEE Antennas and Wireless Propagation Letters, Volume 6, Issue 99, Page(s):525 - 528.

(b) Ebadi, S. \& Forooraghi, K. (2008). Comparative study of annular and rectangular waveguides for application in annular waveguide slot antennas (AWSA), Journal of Electromagnetic Waves and Applications , Vol. 22, 2217-2230.

(c) Ebadi, S. \& Forooraghi, K. (2008). Waveguide Design Considerations in Annular Waveguide Slot Antennas (AWSA), Proceedings of 2008 IEEE Int'll Sym. on Antennas and Prop., 2008, San Diego, USA.

(d) Ebadi, S. \& Forooraghi, K. (2008). Annular Waveguide Analysis for Application in Annular Waveguide Slot Antennas (AWSA), Proceedings of 38th European MW Conf., October 2008, Netherlands.

(e) Ebadi, S. \& Forooraghi, K. (2009). Green's function derivation of an annular waveguide for application in method of moment analysis of annular waveguide slot antennas, Progress In Electromagnetics Research, PIER 89, 101-119.

(f) Ebadi, S. \& Forooraghi, K. (2008). Polarization agility in annular waveguide slot antenna arrays, Proceedings of The 20th Asia-Pacific Microwave Conf. 2008, Hong Kong, China.

(g) Ebadi, S. \& Forooraghi, K. (2009). Linear and circular polarization agility in annular waveguide slot antennas, Proceedings of 2009 IEEE radio and wireless symposium (RWS2009), San Diego, 2009, CA, USA. 


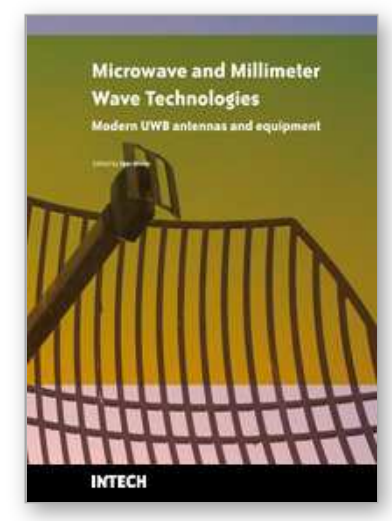

\section{Microwave and Millimeter Wave Technologies Modern UWB antennas and equipment}

Edited by Igor Mini

ISBN 978-953-7619-67-1

Hard cover, 488 pages

Publisher InTech

Published online 01, March, 2010

Published in print edition March, 2010

\section{How to reference}

In order to correctly reference this scholarly work, feel free to copy and paste the following:

Siamak Ebadi and Keyvan Forooraghi (2010). Novel Polarization-Agile Annular Waveguide Slot Antennas, Microwave and Millimeter Wave Technologies Modern UWB antennas and equipment, Igor Mini (Ed.), ISBN: 978-953-7619-67-1, InTech, Available from: http://www.intechopen.com/books/microwave-and-millimeterwave-technologies-modern-uwb-antennas-and-equipment/novel-polarization-agile-annular-waveguide-slotantennas

\section{INTECH}

open science | open minds

\section{InTech Europe}

University Campus STeP Ri

Slavka Krautzeka 83/A

51000 Rijeka, Croatia

Phone: +385 (51) 770447

Fax: +385 (51) 686166

www.intechopen.com

\section{InTech China}

Unit 405, Office Block, Hotel Equatorial Shanghai

No.65, Yan An Road (West), Shanghai, 200040, China

中国上海市延安西路65号上海国际贵都大饭店办公楼 405 单元

Phone: +86-21-62489820

Fax: +86-21-62489821 
(C) 2010 The Author(s). Licensee IntechOpen. This chapter is distributed under the terms of the Creative Commons Attribution-NonCommercialShareAlike-3.0 License, which permits use, distribution and reproduction for non-commercial purposes, provided the original is properly cited and derivative works building on this content are distributed under the same license. 